\title{
TMusic: A Music Player Based on Tangible User Interface
}

\author{
Handan Zhang ${ }^{1, a}$, and Xinglong Gong ${ }^{2, b}$ \\ ${ }^{1}$ Department of Media Arts and Technology, Harbin Institute of Technology, Hei Longjiang Province, \\ Chinese \\ ${ }^{2}$ Department of Media Arts and Technology, Harbin Institute of Technology, Hei Longjiang Province, \\ Chinese
}

azhanghandan0225@163.com, ${ }^{b} x$ lgong0451@163.com

\section{Keywords: Tangible User Interface, Music Player, Object Tracking}

Abstract. Tangible User Interface is a new human-computer interaction interface which purpose is to interact with the digital world through the objects in physical world. Based on this, we present a novel interaction system allow user to play music, and we call it TMusic. In the TMusic, user can manipulate the augmented objects and TMusic will track user's operation to play or change music or change music visual effect. And user can define their augmented objects to represent different music. It's convenient for user to play and enjoy music.

\section{Introduction}

In the era of PC, when we enjoy music, we have to turn on the PC or ipod, then I need to pick the preferred song and press the play button to play, it's quite inconvenient for us to do this. Thanks to the development of speech recognition technology, we now can be easy to speak to our phone "I want music”, The phone will play music automatically for us. But when we want to hear the music which I don't remember the name of it, at this time the phone is helpless, I have to find the music in the folder which is labeled the name I like. Why can’t exist a more personal way to help us to choose the music and use a more convenient way to play music? However, tangible interaction technology provides a potential for such idea.

In recent years, with the development of sensor technology and wearable intelligent device, a new type of interaction becomes more and more popular. People have grown accustomed to sway to control the game character to move. In the WeChat, we can add friends by the function shake. This novel type of interaction is beyond our imagination in the graphical user interface. However, as early as 1997, professor Hiroshi of MIT media group has defined this interaction ${ }^{[1]}$. Tangible User Interface is to contact the digital information in the virtual world with objects in the physical world and we can easily control the digital information in the virtual world by the objects of the physical world. In the definition of Tangible User Interface, the professor Hiroshi explained that the objects are taken as input and output tool, we can interact with the digital in-formation in the virtual world directly.

In this article, the author comes forward with a new type of music player based on the tangible user interface, which is called TMusic, and has explained in details how to implement. In the TMusic, users can move the objects up and down to control the volume, users can move the objects left and right to control the graphic of music, also users can add and reduce the number of objects to choose the music, also uses can choose different emotion model objects to choose music, sad emotion model objects represent sad music, happy emotion model objects represent happy music.

In the TMusic, user can easily define their person-al music player in a convenient way. Fig. 1 shows what the TMusic looks like, there is a workbench for users to move and rotate human model objects. Be-low the workbench there is a camera to capture users operation, and also a projector to display the visual effect on the wall. So when users move the objects on the workbench, the music will change and the image on the wall will also change. 


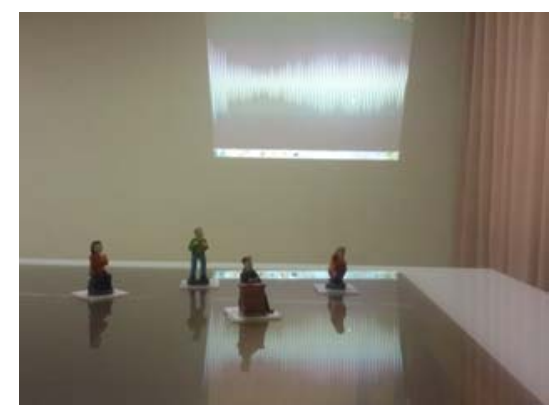

Fig. 1. What it looks like about TMusic.

\section{Characteristic of Tangible User Interface}

In the article "Tangible Bits: Beyond Pixels" [2], Professor Hiroshi has defined the concept of tangible user interface, and summarized the difference between tangible user interface and graphical user interface. In my opinion, tangible user interface has two basic characteristics.

In the physical world, we have learned a lot of experience models, for example, trash is where garbage is placed, and studying room is utilized to study and work. These experience models to ensure our normal life in the physical model. However, in the graphical user interface, we have to learn from these again. We have to remember these different icons representing meanings. We have to learn to operate the software again and again until we master it. So we waste a lot of time and energy to steer it be-fore we are used. However, in the tangible user interface, the process of learning is a lot of easier. In the TMusic, users can put 'sad' model object in the scene, then the player will play 'sad' music, then users can understand different emotion models represent different kind of music, users have learned to how to play music in the TMusic, then users may move the model objects in the scene, the player will response user operation. Through this, users will know how to use TMusic before they read instructions. So in the TMusic, we try our best to make it as simple as possible, and try to join these operations with user's common behavior.

With the development of sensor technology, software designers can be easier to collect various kinds of user's information. In these information, we need to extract the valuable information and utilize it in a right way. Such as GPS, the designers can use it to locate the user's location, so we can easily recommend hotels, airplanes according to user's location information, which can be useful for user's travel. So in the current human-computer interaction technology, it's more and more important to collect user's information. In the tangible user interface, we control the digital information in the virtual world by the objects, we can hide all kinds of sensor devices in the objects' inside, which is a convenient way to collect user's information, such as user's operation, user's strength, of course, we also need stronger algorithm to track and analyze user's behavior in real-time. In the TMusic, we track user's operation, the movement of the objects, the number of the objects, and the rotation of the objects in real-time, so we can be highly responsive to user's operation in real-time.

\section{Implementation}

Object Tracking. In the research of tangible user interface, object tracking is a problem to be taken into account, we can divide it into two small problems, one is the movement of the objects, and another is to know how many objects in the current scene. In the tangible user interface, objects movement is arbitrary. We couldn't be easy to know the movement of the objects, which is more difficult than in the graphical user interface. So in most of tangible user interface system, fixing the movement of objects to 2D plane is a good way to solve the problem, through this, we can reduce the calculation difficult to make our sys-tem more sensitive to response users operation. We call it "Desktop", such as AudioPad ${ }^{[3]}$, Block Jam ${ }^{[4]}$, reacTable ${ }^{[5]}$, these outstanding tangible user interface application are all "Desktop". Based on the same consideration, TMusic is "Desktop", which is a music player, user can be easy to control model objects to select songs, control volume and so on, all the presentation are in the web browser. 
What is different with "Audiopad", "Black Jam” is that users have a right to define their objects in the TMusic, user can choose what they like, what they want to be objects. Those all kinds of self-defined objects will make it different for system to recognize these objects, so in this project, in the TMusic, we come up with a general model which is called "Augmented Objects", these augmented objects will help system to recognize user-defined objects. Binding a special tag information on the objects that the user chooses, through this way, one augmented object is created. TMusic will recognize the tag information to know what it is. Fig. 2 shows how TMusic deals with augmented object $\mathrm{A}$.

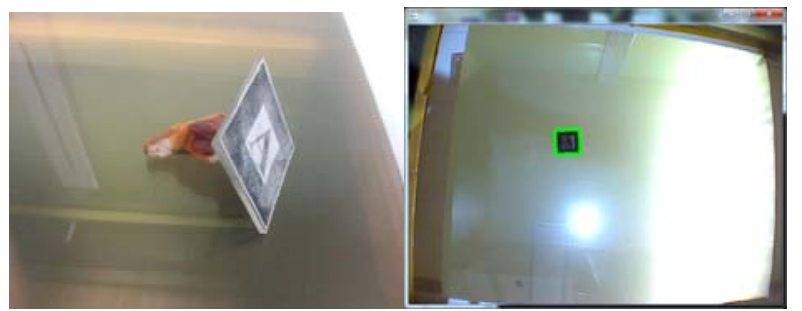

Fig. 2. How TMusic deals with augmented object A

So for a variety number of user-defined objects, users only need to fill the tag information into a con-figuration file, so TMusic can easily identify what it is according to the tag information. By this, TMusic not only knows how many objects in the scene, and also knows what it means. With the help of augmented objects, we change the recognition of the objects to become the recognition of the tag information, which not only reduces the difficulty of calculation, and easy to find out the movement of the objects. In the TMusic, we preset 2 kinds of motion, move up and down, move right and left, which can control the images of the music and the volume of the music, we can also detect the number of augmented objects to choose songs.

Structure of TMusic. Some hardware is needed before we talk about TMusic, we need one usb camera to capture the users' operation. We need a projector to display the visual effect. These hardware acts as input and output tool.

TMusic has 3 components to deal with users' operation, one is to detect the number of augmented objects and the movement of augmented objects. This component is the major component for TMusic, which recognized tag objects. Second component is used to response users' operation, and display music graphic. At last, component exchange data between the first component and the second one. In the Fig. 3, RModel represents recognition system, PModel represents presentation system.

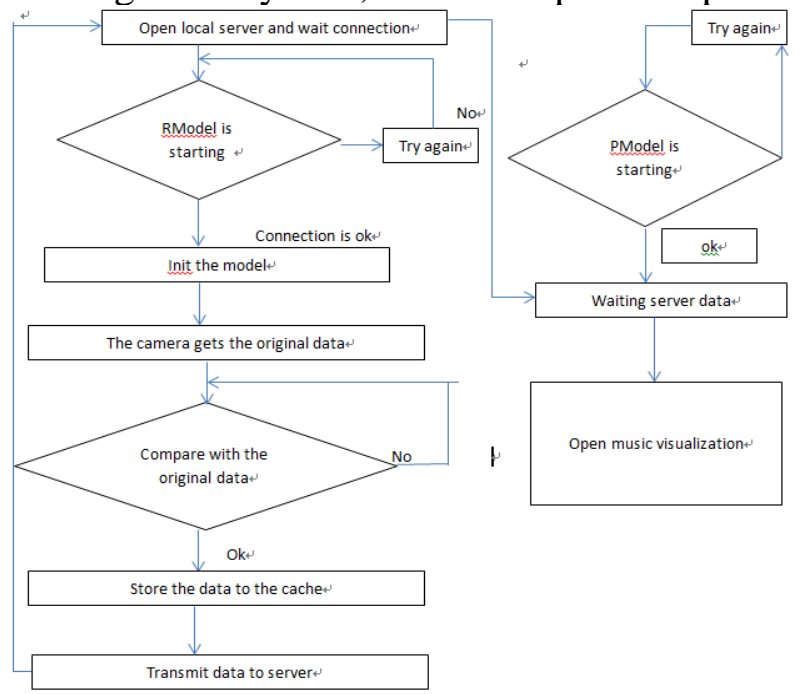

Fig. 3. How three subsystems work together

First, we need to open the server system, which is used to observe the connection, then open presentation and recognition. Then the component will wait users' operation, if users put augmented objects into the scene, the recognition component will detect whether it is the right object, if ok, 
recognition component will pass data to presentation component through server, then display component will display video about the music, which is created by the algorithm in real-time.

Components. In the recognition component, we choose ArtoolKit as our development tool. Built on the ArtoolKit, each time we capture the video, we will compare the original translation and rotation data with the lasted translation and rotation, if there has some difference, we open a thread to pass the data to the server through win32 socket.

In the server, NodeJs is used as the development tool, it's easy to create a local server and easy to deal with the data coming from the recognition system. Once processed, the server system will pass the data to the presentation system. Because of the different transfer protocol between the display and recognition system, a server is used to deal with the data as a middleware.

In the display, the web browser is used as graphic output, in the web browser we can easy to visualize the music because HTML5 provides a lot of application interface for us to deal with the music, and we can also reference a lot of outstanding works about music visualization in GitHub. In the TMusic, I reference surunzi's work "VisualMusic" in the GitHub, and make it more suitable for my work. At last, for better visual effect, I use a projector to show the graphical result.

\section{Summary}

Up to now, I have implemented a music player which has the basic function based on tangible user interface. It can select songs and control volume and visual music and easy to get designed by user-self. What it matters is the case that TMusic frees us from the complicated interface. Maybe TMusic's function is not perfect compared with the music software in the graphical user interface, but it turns out to be that it has potential for us to create a convenient way to enjoy music.

In the future, I want to add more interaction in the TMusic, user can control the visual effect dynamically through the augmented objects, such as, When I play sad music, I hope the color of visual music is darker and the rhythm of the image is slower.

\section{Acknowledgements}

I appreciate my teacher Ou Jian, he gives me a lot of help when I implement the project, and I also appreciate my classmates, they give me a hand a lot. Thank you surunzi, your outstanding work makes me easy to complete music visualization. Thanks to the authors of “AudioPad”, "Block Jam”, "reacTable”, your outstanding works inspire me a lot.

\section{References}

[1] Ullmer, Brygg, and Hiroshi Ishii: The metaDESK: models and prototypes for tangible user interfaces. Proceedings of the $10^{\text {th }}$ annual ACM symposium on User interface software and technology: 223-232 (1997)

[2] Hiroshi Ishii: Tangible bits: beyond pixels. Proceeding of the $2^{\text {nd }}$ International Conference on Tangible and Embedded Interaction: xv-xxv (2008)

[3] Patten, James, Ben Recht, and Hiroshi Ishii: Audiopad: a tag-based interface for musical performance. Proceedings of the 2002 conference on new interfaces for musical expression: 1-6 (2002)

[4] Newton-Dunn, Henry, Hiroaki Nakano, and James Gibson: Block Jam: a tangible interface for interactive music. Proceedings of the 2003 conference on new interfaces for musical express: 170-177 (2003)

[5] Jordà, Sergi, Günter Geiger, Marcos Alonso, and Martin Kal-tenbrunner: The reacTable: exploring the synergy be-tween live music performance and tabletop tangible inter-faces. In Proceedings of the 1st International Conference on Tangible and Embeded Interaction: 139-146 (2007) 